\title{
Application Article \\ Impact of Ship Motions on Maritime Radio Links
}

\author{
William Hubert, ${ }^{1}$ Yvon-Marie Le Roux, ${ }^{2}$ Michel Ney, ${ }^{3}$ and Anne Flamand ${ }^{1}$ \\ ${ }^{1}$ Communications Department, DCNS, France \\ ${ }^{2}$ Electronic Department and Labsticc, Telecom Bretagne, France \\ ${ }^{3}$ Microwaves Department and Labsticc, Telecom Bretagne, France \\ Correspondence should be addressed to William Hubert, william.hubert@telecom-bretagne.eu
}

Received 27 July 2012; Revised 22 October 2012; Accepted 7 November 2012

Academic Editor: Thomas Kürner

Copyright (๑) 2012 William Hubert et al. This is an open access article distributed under the Creative Commons Attribution License, which permits unrestricted use, distribution, and reproduction in any medium, provided the original work is properly cited.

The improvement of maritime radio links often requires an increase of emitted power or receiver sensitivity. Another way is to replace the poor antenna gains of traditional surface ship whips by novel antenna structures with directive properties. However, ship motions, especially when launches are involved, may affect the radio link quality. Therefore, a tool for modelling the impact of ship motions on such antenna structures was developed. It helps the specification of antenna radiating parameters and improves the radio link performance evaluation. This tool includes a deterministic two-ray model for radio-wave propagation over the sea surface. The geometrical 3D engine for ship motions has the advantage to be compatible with any propagation model and antenna structure.

\section{Introduction}

This paper presents the development of a deterministic transmission channel model for the improvement of a high data rate $\left(>10 \mathrm{Mbits} \cdot \mathrm{s}^{-1}\right)$ radio-link in the S-band ( 2 to $4 \mathrm{GHz}$ ). This link is supposed to reach the largest possible range between small surface ships. First, the link budget at the approach of the radio horizon by means of the ITU P.526 model [1] was computed. It shows that establishing such a link between ships requires a huge increase in emitted power if poor antenna gains (about $2 \mathrm{dBi}$ ) are used. Hence, the purpose of the transmission model is to help the specification of novel antenna structures with gains above $10 \mathrm{dBi}$. To reproduce the real situation as accurately as possible, especially when ship motions are relevant, a 3D geometrical model was developed. The computational engine can accommodate any antenna and propagation model. However, a simple two-ray model and dipole antennas are used in this paper for illustrative example.

After a description of the propagation model, a section is then devoted to the presentation of the geometrical context for the integration of ship motions. The next section is dedicated to the computation of the maximum antenna angular deviation for given sea-wave data. Then, the integration of antenna radiating parameters is explained. Finally, an expression of the received power is derived using channel matrix formalism, followed by a presentation of simulated results.

\section{Propagation Model}

The propagation is described by a two-ray model that includes a direct path between the transmitter $(A)$ and the receiver $(B)$ and a path coherently reflected (in $P$ ) by the sea surface (Figure 1). This model has recently been confirmed in [2] by measurements. It is applied here on a spherical earth of effective radius $R_{e}$ which corresponds to a mean atmospheric refractive index. The coherently reflected path is obtained according to the Snell-Descartes law and its amplitude is primarily determined by the Fresnel reflection coefficients:

$$
\rho_{\|}=\frac{\sin \varphi-(1 / \eta) \sqrt{\eta-\cos ^{2} \varphi}}{\sin \varphi+(1 / \eta) \sqrt{\eta-\cos ^{2} \varphi}}
$$




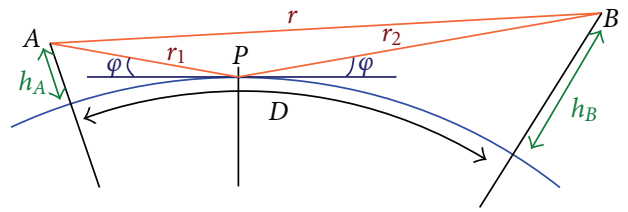

FIgURE 1: 2D geometry of the two-ray model.

for the vertical (or electric field parallel to the incidence plane) polarization,

$$
\rho_{\perp}=\frac{\sin \varphi-\sqrt{\eta-\cos ^{2} \varphi}}{\sin \varphi+\sqrt{\eta-\cos ^{2} \varphi}}
$$

for the horizontal (or orthogonal to the incidence plane) polarization.

In the above expressions, $\eta$ stands for the relative complex permittivity of the sea water surface. It can be defined by the Debye formula applied by Stogryn in [3].

The reflected energy is also affected by a divergence factor which produces a larger aperture of the beam after reflection. Its expression is given by Beckmann and Spizzichino in [4] and depends on geometric parameters previously defined in Figure 1:

$$
\mathrm{FD}=\left[1+\frac{2 r_{1} r_{2}}{R_{e}\left(r_{1}+r_{2}\right) \sin \varphi}\right]^{-1 / 2}\left[1+\frac{2 r_{1} r_{2}}{R_{e}\left(r_{1}+r_{2}\right)}\right]^{-1 / 2} .
$$

The model validity is primarily bounded by the variations of FD. This factor must not be used after a limiting distance which corresponds to the transition between the two-ray model and the ITU P.526 model. An attempt of integrating the latter does not give enough satisfaction with regard to the transition range. Moreover, the program objective is to assist the antenna specification. So, the design of the link is done at long range by means of the ITU model. Comparison between these different models is shown in Figure 2, obtained at $2.4 \mathrm{GHz}$, with an effective isotropic radiated power (EIRP) of 1 Watt, with both antennas at 6 meters above the sea surface.

Expected results are obtained in Figure 2 since good vertical and poor horizontal spatial diversities are observed. Besides, it appears worthless to take benefits from the polarization diversity observed at short ranges because of the unfavorable two-ray recombination in horizontal polarization.

Finally, a second factor is applied to the specularly reflected energy in presence of sea waves. If a normal distribution hypothesis of sea surface elevations is made, the reduction factor $\rho_{r}$ depends on their standard deviation $\sigma$. According to Miller et al. [5], the reduction factor is given by the following expression:

$$
\rho_{r}=\exp \left[-2(2 \pi g)^{2}\right] \cdot I_{0}\left[2(2 \pi g)^{2}\right]
$$

in which

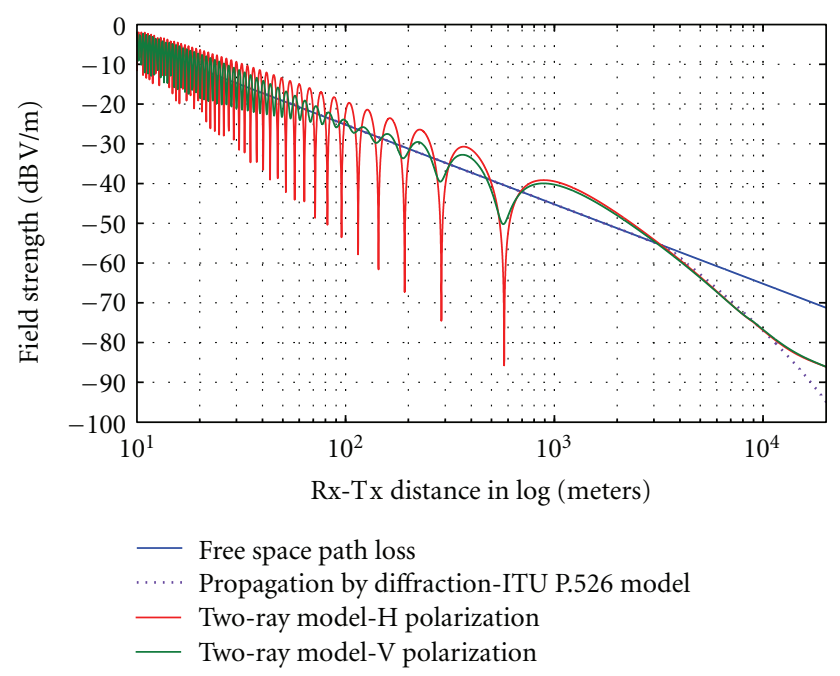

FIgURE 2: Field strength (dB V/m) versus distance (log scale).

(i) $g=(\sigma \sin \varphi) / \lambda$ is the Rayleigh criterion,

(ii) $I_{0}[\cdot]$ is the modified Bessel function of the first kind and order zero, and

(iii) $\lambda$ is the EM wavelength in meters.

Figure 3 is obtained with the same parameters as before but for a sea state 3 on Douglas sea scale $(\sigma=0.5$ meters). A slight swell is sufficient to significantly reduce the coherently reflected energy. The result is a partial vanishing of fading figures. The energy which is no longer coherently reflected leads to a diffuse scattering. However, diffusion is not yet integrated in this deterministic model. Further improvements could be made by introducing that diffuse component in the propagation model. A good starting point would be the analysis proposed in [6] by Karasawa and Shiokawa.

\section{Integration of Ship Motions}

The previously defined $2 \mathrm{D}$ geometry has to evolve to three dimensions to integrate ship motions. A global coordinate system $(O, \vec{X}, \vec{Y}, \vec{Z})$, in which $O$ is the Earth center, is shown in Figure 4. Angle $\alpha$ is defined according to the Intership distance $D$ (Figure 1 ) and $\beta$ stands for their relative orientation.

All coordinates will be further expressed in this global system by means of translation vectors and rotation matrices. Then, a local coordinate system attached to each ship center of mass is defined wherein ship motions are described (Figure 5).

Regarding the ship motions, one shall define below the dedicated terms related to the local coordinate system:

(i) heave is a translation along $\vec{z}$ axis (vertical motion),

(ii) roll is a rotation about $\vec{x}$ axis,

(iii) pitch is a rotation about $\vec{y}$ axis.

The above motions can be simply described by sinus functions. Therefore, they are characterized here by their 


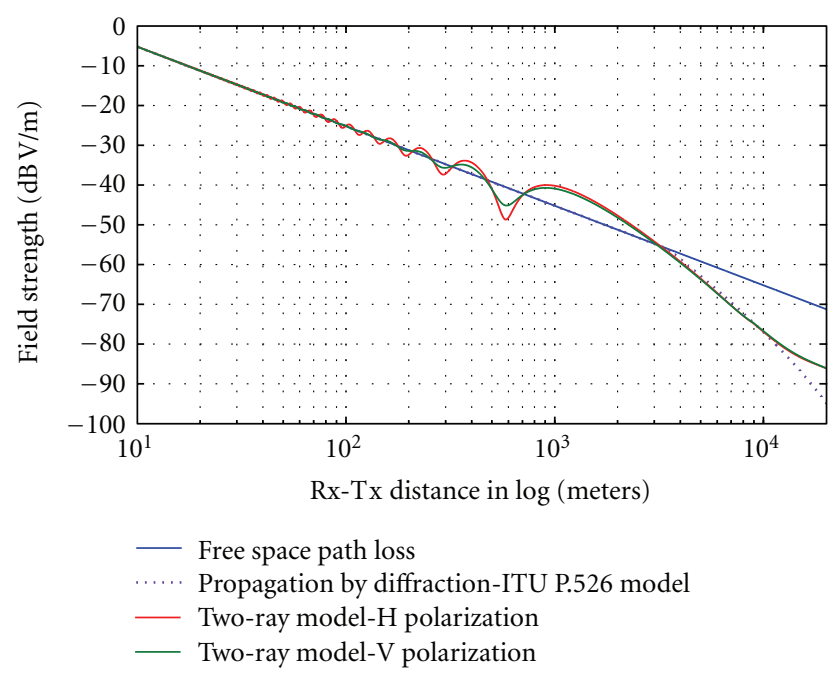

FIGURE 3: Field strength ( $\mathrm{dB}$ V/m) versus distance (log scale), sea state 3 on Douglas scale.

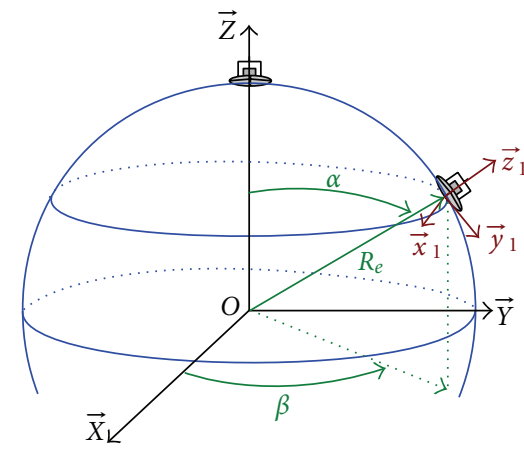

Figure 4: Global coordinate system for ship positions.

amplitude and period only. Further improvements could be made by introducing recorded data of real ship motions. Antenna locations are also described in this system by their onboard coordinates with a proper orientation. Finally, a coordinate system attached to the antenna center $(A)$ is introduced according to antenna measurements or simulations (Figure 6).

By expressing all geometric objects in the global coordinate system, antenna position and velocity, rays arrival, and departure angles can be determined at any time.

\section{Maximum Angular Deviation}

Without real ship motion records, approximate geometrical relations can be derived for antenna maximum angular deviation from vertical direction. Knowing the maximum crest-to-trough wave height $H_{\max }$ and the wavelength $\lambda_{s}$, one can evaluate the maximum antenna angular deviation:

$$
\theta_{\max }=\arcsin \left(\frac{\pi H_{\max }}{\sqrt{\lambda_{s}^{2}+\pi^{2} H_{\max }^{2}}}\right) .
$$

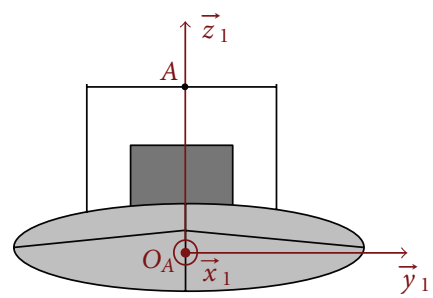

FIgURE 5: Local coordinate system for the ship in $A$, front view of the ship.

Several simulations have shown that this angular deviation represents the maximum deviation from the position at rest for distances above 100 meters. For linearly polarized antennas, it also leads to a maximum polarization loss factor (PLF) given by

$$
\mathrm{PLF}_{\mathrm{dB}}=20 \log _{10}\left(\cos 2 \theta_{\max }\right) .
$$

Using the fundamental mode of the Pierson-Moskowitz spectrum for fully developed regular wind waves [7], a complete data set is defined for the studied case. It includes the period $T_{s}$ and the standard deviation of sea-wave elevations $\sigma$ :

$$
\begin{array}{rlrl}
H_{\max } & \simeq 5.7 \mathrm{~m}, & T_{s} \simeq 9.2 \mathrm{~s}, \\
\lambda_{s} \simeq 131.4 \mathrm{~m}, & \sigma \simeq 1 \mathrm{~m} .
\end{array}
$$

What is introduced in (5) leads to

$$
\theta_{\max }=7.6^{\circ} .
$$

Note that the result on $\theta_{\max }$ remains approximately constant for any $H_{\max }$. This is due to the fully developed regular wind wave assumption. However, if both $H_{\max }$ and $\lambda_{s}$ are determined by any means (models or measurements), (5) still holds for small ships.

\section{Antenna Integration}

A radiation vector function $\mathbf{F}$ is introduced to account for polarisation state and antenna gain along any direction. As in [8], $\mathbf{F}$ depends on the realized gain (which integrates impedance mismatch) for a given set of departure (emitting antenna) or arrival (receiving antenna) angles:

$$
\mathbf{F}=\sqrt{G(\chi, \psi)} \mathbf{U}=\sqrt{G(\chi, \psi)}\left(\begin{array}{c}
U_{\chi}(\chi, \psi) \\
U_{\psi}(\chi, \psi)
\end{array}\right) .
$$

In the above expression, $\chi$ and $\psi$ stand for the elevation angle and the azimuth angle, respectively. $\mathbf{U}$ is a normalized vector which corresponds to the complex ratio (amplitude and phase) emitted (or received) along $\vec{u}_{\chi}$ and $\vec{u}_{\psi}$ components (see Figure 6).

A database was created for the simulations. It consists of multiple tables of data extracted from electromagnetic (EM) simulators, in our case HFSS. These tables represent 


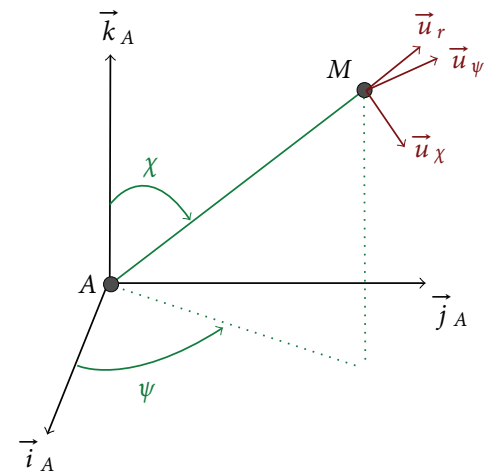

Figure 6: Antenna coordinate system with unit vectors for field components.

realised gain and antenna field components as expressed in (9). Finally, for wide band communications, the radiation vector function database $\mathbf{F}$ would have to be generated for several frequencies.

\section{Expression of the Received Power}

First of all, a channel matrix is built by using the unit vectors (see Figure 6) expressed in the same coordinate frame. It expresses intrinsically the PLF. Then, the sphericaldivergence and phase terms are applied to obtain the directpath channel matrix:

$$
\mathbf{C}_{\mathrm{DP}}=\frac{1}{r}\left(\begin{array}{lll}
\vec{u}_{\chi}^{A} \cdot \vec{u}_{\chi}^{B} & \vec{u}_{\psi}^{A} \cdot \vec{u}_{\chi}^{B} \\
\vec{u}_{\chi}^{A} \cdot \vec{u}_{\psi}^{B} & \vec{u}_{\psi}^{A} \cdot \vec{u}_{\psi}^{B}
\end{array}\right) e^{-j 2 \pi r / \lambda}
$$

Radiation vector functions for the transmitting antenna $\mathbf{F}^{A}(\chi, \psi)$ and for the receiving antenna $\mathbf{F}^{B}(\chi, \psi)$ are then applied to both sides of this matrix to obtain a transmission scalar $s_{\text {DP }}$. Thus, with (9) and (10),

$$
\begin{aligned}
s_{\mathrm{DP}}= & \frac{1}{r} \sqrt{G^{A}} \sqrt{G^{B}}\left(\begin{array}{cc}
U_{\chi}^{B} & U_{\psi}^{B}
\end{array}\right) \\
& \times\left(\begin{array}{rr}
\vec{u}_{\chi}^{A} \cdot \vec{u}_{\chi}^{B} & \vec{u}_{\psi}^{A} \cdot \vec{u}_{\chi}^{B} \\
\vec{u}_{\chi}^{A} \cdot \vec{u}_{\psi}^{B} & \vec{u}_{\psi}^{A} \cdot \vec{u}_{\psi}^{B}
\end{array}\right)\left(\begin{array}{c}
U_{\chi}^{A} \\
U_{\psi}^{A}
\end{array}\right) e^{-j 2 \pi r / \lambda} .
\end{aligned}
$$

For the coherently reflected path, unit vectors $\vec{u}_{\chi, \psi}^{A, B}$ have to be decomposed into a parallel $\vec{u}_{\|}$and an orthogonal $\vec{u}_{\perp}$ components to the sea surface as shown in Figure 7.

Then, one can define the reflection dyadic $\overline{\overline{\mathbf{R}}}$ :

$$
\overline{\overline{\mathbf{R}}}=\mathrm{FD} \cdot \rho_{r} \cdot\left(\begin{array}{cc}
\rho_{\|} & 0 \\
0 & \rho_{\perp}
\end{array}\right)
$$

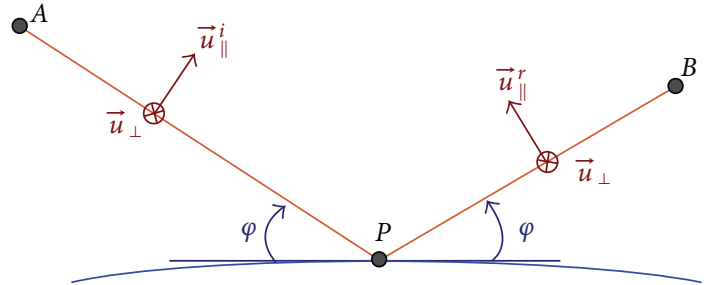

Figure 7: Unit vector definition for reflection.

in which $\rho_{\|}, \rho_{\perp}, \mathrm{FD}$, and $\rho_{r}$ are defined in (1), (2), (3), and (4), respectively. Thus, the propagation channel matrix for the reflected path becomes

$$
\begin{aligned}
\mathrm{C}_{\mathrm{RP}}= & \frac{1}{r_{1}+r_{2}}\left(\begin{array}{ll}
\vec{u}_{\chi}^{B} \cdot \vec{u}_{\|}^{r} & \vec{u}_{\chi}^{B} \cdot \vec{u}_{\perp} \\
\vec{u}_{\psi}^{B} \cdot \vec{u}_{\|}^{r} & \vec{u}_{\psi}^{B} \cdot \vec{u}_{\perp}
\end{array}\right) \\
& \times \overline{\overline{\mathbf{R}}}\left(\begin{array}{cc}
\vec{e}_{\chi}^{A} \cdot \vec{u}_{\|}^{i} & \vec{u}_{\psi}^{A} \cdot \vec{u}_{\|}^{i} \\
\vec{e}_{\chi}^{A} \cdot \vec{u}_{\perp} & \vec{u}_{\psi}^{A} \cdot \vec{u}_{\perp}
\end{array}\right) e^{-j 2 \pi\left(r_{1}+r_{2}\right) / \lambda} .
\end{aligned}
$$

The same approach used to obtain (11) is applied to derive the second scalar for the coherently reflected path:

$$
\begin{aligned}
s_{\mathrm{RP}}= & \frac{1}{r_{1}+r_{2}} \sqrt{G^{A}} \sqrt{G^{B}}\left(\begin{array}{ll}
U_{\chi}^{B} & U_{\psi}^{B}
\end{array}\right)\left(\begin{array}{ccc}
\vec{u}_{\chi}^{B} \cdot \vec{u}_{\|}^{r} & \vec{u}_{\chi}^{B} \cdot \vec{u}_{\perp} \\
\vec{u}_{\psi}^{B} \cdot \vec{u}_{\|}^{r} & \vec{u}_{\psi}^{B} \cdot \vec{u}_{\perp}
\end{array}\right) \\
& \times \overline{\overline{\mathbf{R}}}\left(\begin{array}{cc}
\vec{u}_{\chi}^{A} \cdot \vec{u}_{\|}^{i} & \vec{u}_{\psi}^{A} \cdot \vec{u}_{\|}^{i} \\
\vec{u}_{\chi}^{A} \cdot \vec{u}_{\perp} & \vec{u}_{\psi}^{A} \cdot \vec{u}_{\perp}
\end{array}\right)\left(\begin{array}{c}
U_{\chi}^{A} \\
U_{\psi}^{A}
\end{array}\right) e^{-j 2 \pi\left(r_{1}+r_{2}\right) / \lambda} .
\end{aligned}
$$

Note that the unit vectors $\vec{u}_{\chi, \psi}^{A, B}$ and radiation vector functions in (11) and (14) are not the same because antenna polarization and gain are subject to change with the propagation direction. Finally, using classical formulas, one can evaluate the received power $P_{r}$ as a function of the supplied power at antenna terminals $P_{f}$ :

$$
P_{r}=\left(\frac{\lambda}{4 \pi}\right)^{2} P_{f}\left|s_{\mathrm{DP}}+s_{\mathrm{RP}}\right|^{2}
$$

in which $s_{\mathrm{DP}}$ and $s_{\mathrm{RP}}$ are given by (11) and (14), respectively. In the above expressions, time (related to ship motions) and frequency (for electromagnetic wave propagation) are involved.

\section{Simulated Results}

A simulation with a transmitter which undergoes the previously defined motions (sea state 5 on Douglas scale) and a receiver ashore (motionless) is proposed. Both antennas are at six meters above the sea surface with a distance of 700 meters between them. As shown in Figure 2, this range naturally leads to a ray recombination equal to the freespace path loss. Both antennas are dipoles with maximum gain of $2.1 \mathrm{dBi}$. Variations of the received power versus time are shown in Figure 8. Different simulations have 


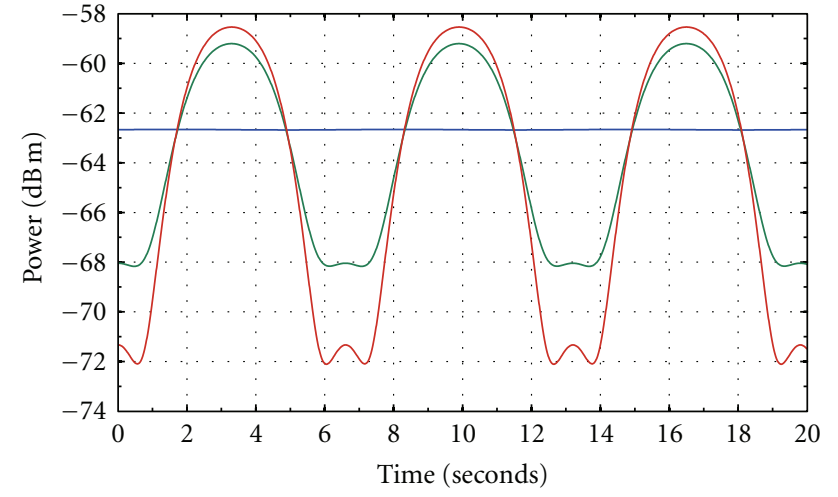

_ Free space path loss

— Two-ray model-vertical dipoles

— Two-ray model-horizontal dipoles

FIGURE 8: Received power versus time, sea-state 5, ship motions in the incidence plane.

shown that those variations are mainly due to the tworay recombination which changes with movements. On the other hand, significant losses are observed if antennas with relatively high directivity are used. To illustrate this, Figure 9 shows the gain at transmission for the direct path and for antenna arrays which have higher directivity. Note that similar results are obtained for the reflected path. The maximum gain of the linear vertical array of 8 dipoles is greater than $9 \mathrm{dBi}$, but its half-power beam aperture angle is less than $2 \theta_{\max }$. As a result, instantaneous effective gain fluctuates to values below the ones obtained by linear vertical array of 4 dipoles. This confirms that the $-3 \mathrm{~dB}$ elevation aperture angle has to be at least in the order of $2 \theta_{\max }$ (given by (5)) to preclude large gain reductions. Note that ship motions in the azimuth plane have negligible impact on the gain variation.

Finally, several simulations show that ship motions lead to a polarization loss factor less (PLF) than $3 \mathrm{~dB}$. This indicates that using circular polarization does not bring any advantage because of the deep fadings produced by horizontal polarization (see Figure 2).

\section{Conclusion}

The description of a maritime radio link channel simulator was presented. It includes a two-ray propagation model, antenna parameters, and three-dimensional (3D) ship motion model. Each component of the simulator is independent as the ship motion calculation engine can be used with any propagation model or antennas. The objective of the simulator is to provide a reliable tool for antenna specification and channel performance evaluation. First results show that classical behaviour of the two-ray model remains the main contribution to the channel characteristics if the condition on the maximum deviation angle $\theta_{\max }$ is fulfilled. Antenna $-3 \mathrm{~dB}$ elevation beam aperture has to be chosen according to ship motions, given a radiolink-operational sea state. In addition, the use of vertically

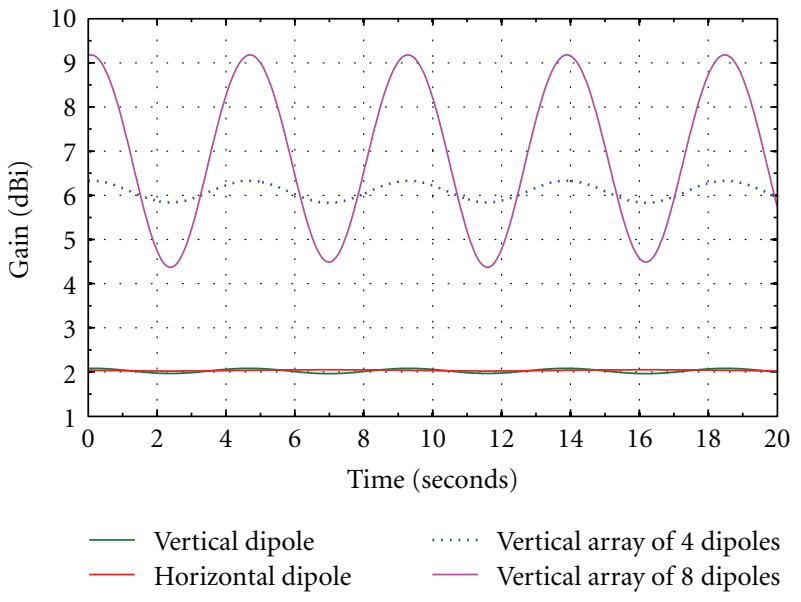

Figure 9: Gains at transmission on the direct path with dipole and arrays of dipoles.

polarized antenna seems to be the most advantageous option. The maximum deviation angle expression is provided and, for small-ship motions, both the polarisation loss factor and the decrease in antenna gain can be predicted. For larger ships, the only parameter that has to be known is the maximum angular deviation between roll and pitch.

\section{Conflict of Interests}

The authors declare that there is no conflict of interests with ANSYS, editor of HFSS.

\section{Acknowledgments}

This work has been done with the support of DCNS and Telecom Bretagne, Mines-Telecom Institute.

\section{References}

[1] ITU Rec. P. 526-12, “Propagation by diffraction," ITU, Geneva, Switzerland, 2012.

[2] Y. M. Le Roux, J. Ménard, C. Toquin, J. P. Jolivet, and F. Nicolas, "Experimental measurements of propagation characteristics for maritime radio links," in Proceedings of the 9th International Conference on Intelligent Transport Systems Telecommunications (ITST '09), pp. 364-369, October 2009.

[3] A Stogryn, "Equations for calculating the dielectric constant of saline water," IEEE Transactions on Microwave Theory and Techniques, vol. 19, no. 8, pp. 733-736, 1971.

[4] P. Beckmann and A. Spizzichino, The Scattering of Electromagnetic Waves From Rough Surfaces, Artech House, 1963.

[5] A. R. Miller, R. M. Brown, and E. Vegh, "New derivation for the rough-surface reflection coefficient and for the distribution of sea-wave elevations," IEE Proceedings $H$, vol. 131, no. 2, pp. 114-116, 1984.

[6] Y. Karasawa and T. Shiokawa, "Simple prediction method for L-band multipath fading in rough sea conditions," IEEE Transactions on Communications, vol. 36, no. 10, pp. 10981104, 1988. 
[7] L. Gardenal, B. Philibert, and R. M. Turner, "Study of the interaction of electromagnetic waves on a sea surface: improvement of the algorithm of low-altitude tracking of radar targets," in Proceedings of the Canadian Conference on Electrical and Computer Engineering, vol. 2, pp. 693-696, 1994.

[8] B. Uguen, L. M. Aubert, and F. T. Talom, "A comprehensive MIMO-UWB channel model framework for ray tracing approaches," in Proceedings of the IEEE International Conference on Ultra-Wideband (ICUWB '06), pp. 231-236, September 2006. 

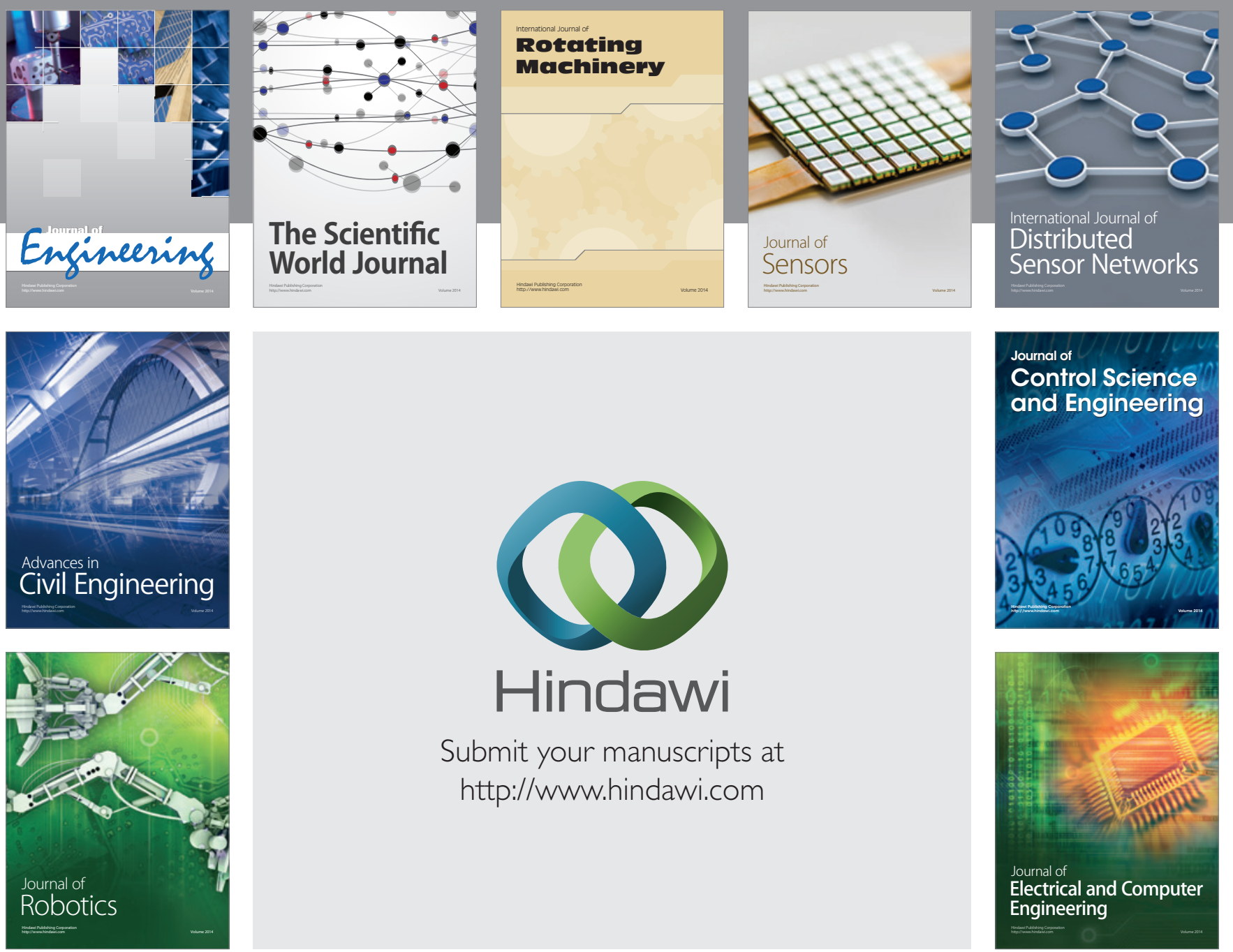

Submit your manuscripts at

http://www.hindawi.com
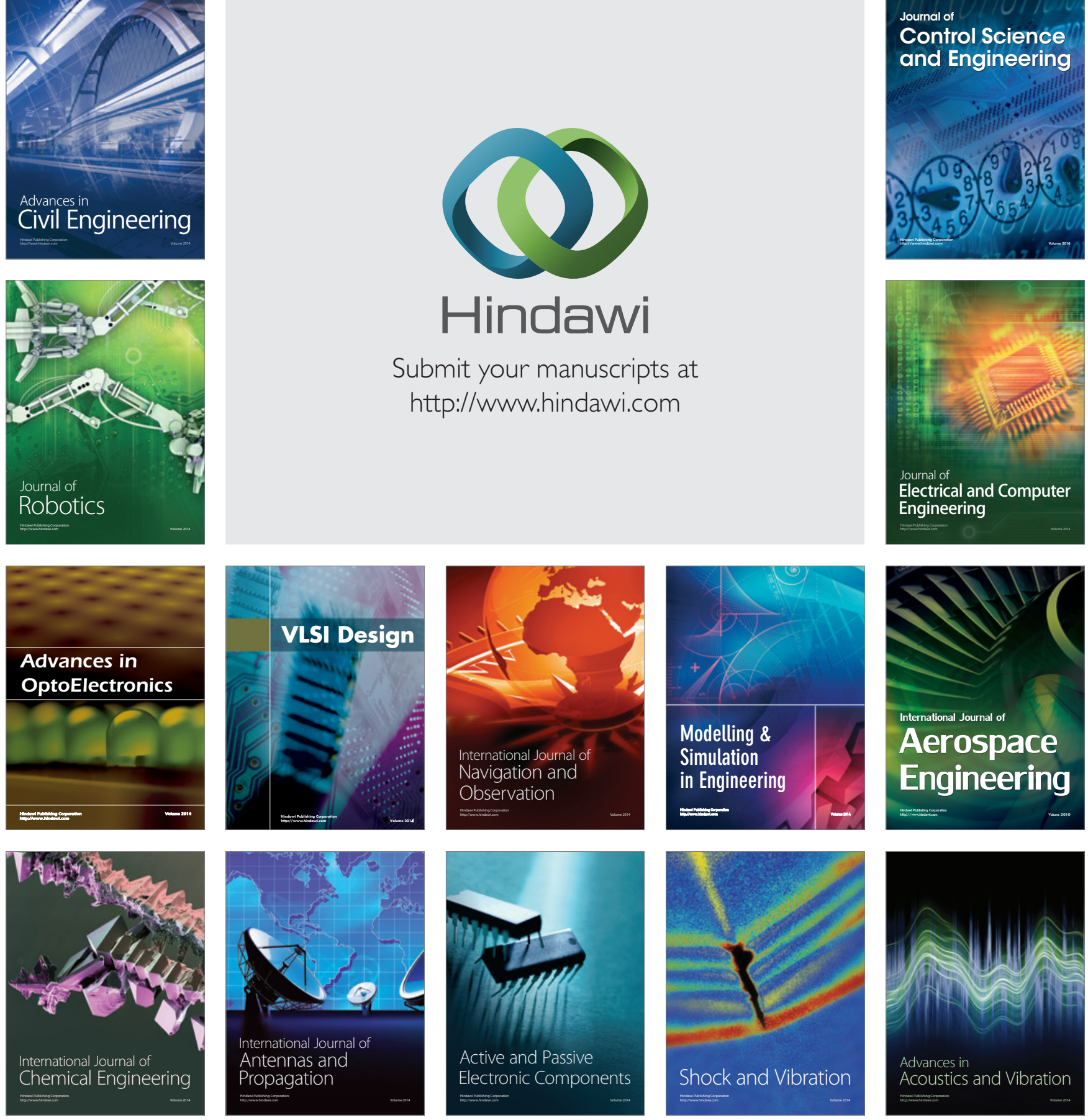